\title{
SORTIR DE L'UTOPIE DE LA COMMUNICATION : UNE APPROCHE ETHNOGRAPHIQUE DES NTIC
}

\author{
Rossella Magli et Yves Winkin' ${ }^{1}$
}

\section{Introduction}

Les historiens des idées devront un jour décrire le déploiement de la notion de communication au cours de la seconde moitié du $\mathrm{XX}^{\mathrm{e}}$ siècle et s'interroger sur les raisons qui ont permis à cette notion issue du sens commun à la fois de gagner une place respectable au sein de disciplines aussi différentes que la linguistique, l'éthologie et la science politique, de polariser une nouvelle configuration scientifique autour d'elle, souvent appelée en France "sciences de la communication et de l'information" (SIC) et, enfin et surtout, de fonder une vision radieuse de l'avenir, reposant sur une circulation toujours plus rapide de l'information, hier grâce aux médias, aujourd'hui grâce aux "Nouvelles Technologies de l'Information et de la Communication" (NTIC).

\footnotetext{
1 Laboratoire d'Anthropologie de la Communication de l'Université de Liège.
}

Recherches en communication, $\mathrm{n}^{\circ} 12,(1999)$. 
Nous voudrions montrer dans cet article que si l'on change de définition de la communication, un recadrage s'opère, qui permet d'analyser autrement les phénomènes sociaux -dont les usages des NTIC- et de démonter ainsi cette "utopie de la communication", qui devient inquiétante à bien des égards, notamment quant à l'ampleur de sa diffusion et à la croyance en son inéluctabilité.

Philippe Breton a proposé dans L'utopie de la communication ${ }^{1}$ une thèse extrêmement séduisante. Au sortir de la Seconde Guerre mondiale, la cybernétique de Norbert Wiener a diffusé un message d'espoir : une société fondée sur la communication, c'est-à-dire sur la transparence, serait la meilleure garantie contre une société totalitaire, dont le mode de fonctionnement repose sur le secret, la dissimulation, la non-communication. Pour Breton, cette vision utopique de la communication est encore bien présente aujourd'hui, notamment au sein du discours sur les NTIC ${ }^{2}$. Nous voudrions montrer qu'il peut ne pas en être ainsi : il suffit de repartir d'une autre perspective, minoritaire mais active, que Breton n' avait pas envisagée. Celle que Sigman ${ }^{3}$ et Leeds-Hurwitz $z^{4}$ appellent "approche sociale de la communication", celle que Winkin ${ }^{5}$ a diffusé en France sous l'expression "Nouvelle Communication". Nous allons y revenir.

Ce que Ph. Breton n'a sans doute pas assez souligné, c'est que la vision wienerienne repose sur une acception commune de communication -mais une acception qui prend des allures savantes. Autant dans le très abstrait Cybernetics or Control and Communication in the Animal and the Machine ${ }^{6}$ de 1948 que dans le très accessible Human Use of Human Beings : Cybernetics and Society ${ }^{7}$, Wiener emploie communication dans le sens de transmission. Certes Wiener s'interroge sur le phénomène :

I Ph. Breton, L'utopie de la communication, Paris, La Découverte, 1992.

2 Ibidem, p. 133.

3 St. Srgman, A Perspective on Social Communication, Lexington (Mass), Lexington Books, 1987.

4 W. LeEds-Hurwitz, Communication in Everyday Life : a Social Interpretation, Norwood (NJ), Ablex, 1989.

5 Y. WINkIN, éd., La nouvelle communication, Paris, Éd. du Seuil, 1981.

6 N. WIEnER, Cybernetics or Control and Communication in the Animal and the Machine, Paris, Hermann, 1948.

7 IDEM, The Human Use of Human Beings : Cybernetics and Society, Boston, Unwin, 1950. Trad. fr. Cybernétique et société. L'usage humain des êtres humains, Paris, U.G.E., 1971. 
Qu'est-ce donc que cette communication qui caractérise l'homme en tant qu'homme? Je consacrerai la plus grande partie de cet ouvrage à l'exposé des théories et des concepts qui contribuent à résoudre ce problème ${ }^{1}$.

Mais la théorie de la communication qu'il propose est avant tout celle qui provient du livre de Claude Shannon et Warren Weaver, The Mathematical Theory of Communication ${ }^{2}$. On le sait, Claude Shannon n'avait aucune ambition théorique pour communication ; c'est pour lui un simple synonyme de transmission, encore une fois. Mais Warren Weaver, chargé d'introduire les articles "redoutablement scientifiques" de Claude Shannon en langage plus simple, s'est emparé du mot et en a fait la clé de voûte d'une "théorie" générale du comportement humain :

Le mot communication sera utilisé ici dans un sens très large incluant tous les procédés par lesquels un esprit peut en influencer un autre. Cela, bien sûr, comprend non seulement le langage écrit ou parlé, mais aussi la musique, les arts plastiques, le théâtre, la danse et, en fait, tout comportement humain ${ }^{3}$.

L'impact de la simplification et de l'amplification des pages très abstraites de Shannon par Weaver sera énorme. D'une part, son introduction va rendre au sens commun des termes qui en avaient été extraits, mais en les lestant d'une aura nouvelle. Ainsi, information, un terme technique qui exprime la mesure de l'incertitude d'un message, devient un synonyme savant et "envoûtant" d'informations (au pluriel) sans référent précis. D'autre part, elle va donner l'illusion que des termes du sens commun ont reçu un pouvoir d'analyse extrêmement vaste -c'est le cas de communication.

Certes Wiener pouvait se dispenser de cette préface de Weaver -il était certainement capable de lire "dans le texte" les formules mathématiques de son ancien élève Shannon. Mais son zèle "missionnaire" l'a amené à adopter la posture de Weaver : souffle, vision, inspiration.

1 Ibid., p. 30.

$2 \mathrm{Cl}$. SHANNON et W. WEAVER, The Mathematical Theory of Communication, Urbana (Ill.) University of Illinois Press. Trad. fr., La théorie mathématique de la communication, Paris, Retz-CEPL, 1975.

3 Ibid., p. 31. 
Après une éclipse dans les années 60-70, la vision wienerienne de la communication est revenue en force sur le devant de la scène publique lors de l'émergence des NTIC. L'expression étrange mais incontournable de société de l'information, les publications officielles de l'Union européenne sur celle-ci (dont le Rapport Bangemann, et ses plus récents développements, sont sans doute l'exemple le plus achevé) et les discours tantôt effrayés (style Monde Diplomatique), tantôt enthousiasmés (style Plan Allègre/Royal) sur les NTIC, sont autant d'indices montrant que la communication -c'est-à-dire la transmission d'informations (au pluriel)- est présentée, comme en 1945-50, comme une valeur progressiste, qui fera advenir une société meilleure et plus juste. La communication est bien l'utopie de la seconde moitié du $\mathrm{XX}^{\mathrm{e}}$ siècle.

\section{Une approche alternative}

Tandis que la vision "télégraphique" commençait à se diffuser aux États-Unis et en Europe, divers chercheurs, dont, paradoxalement, Wiener lui-même, vont construire, notamment sur la base des Macy Conferences sur la cybernétique ${ }^{1}$, une vision très différente de la communication, à la fois systémique, plurielle et multimodale. Cette conceptualisation "orchestrale" de la communication, déjà pressentie avant 1940 par Ch. Cooley, E. Sapir et G.-H. Mead s'est poursuivie tout au long des années 60 et 70 , avec G. Bateson, R. Birdwhistell, A. Scheflen. L'ouvrage de P. Watzlawick, J. Beavin et D. Jackson, Pragmatics of Human Communication ${ }^{2}$ en reformulera certains principes de base dans une perspective d'intervention psychothérapeutique : c'est ce qu'on a appelé en France (l'expression est inconnue aux États-Unis) "l'École de Palo Alto".

Mais cette pensée de la communication peut aussi s'articuler sur une démarche ethnographique et donner lieu à une "anthropologie de la communication ${ }^{3}$. C'est ici que nous troquons notre toge d'historiens des SIC pour une tenue de terrain.

1 St. HeIMs, The Cybernetics Group, Cambridge (Mass.), The MIT Press, 1991.

2 P. WATZlawick, J. BEAVIN et D. JACKSON, Pragmatics of Human Communication, New York, Norton. Trad. fr. Une logique de la communication, Paris, Éd. du Seuil, 1972.

3 Y. Winkin, Anthropologie de la communication. De la théorie au terrain, Bruxelles, De Boeck, 1996. 
Notre projet a en effet consisté à séjourner aussi longtemps que possible dans différentes écoles primaires et secondaires d'Europe et à y observer ce que les acteurs en présence font avec les ordinateurs mis à leur disposition ${ }^{1}$.

Notre cadrage théorique et méthodologique était différent de celui de la plupart des études d'usage des NTIC en milieu scolaire pour deux raisons au moins. D'une part, nous avons voulu travailler sur des rapports collectifs à l'ordinateur et non sur des rapports individuels; non des interactions entre un enfant et un ordinateur mais des interactions entre des classes et les ordinateurs mis à leur disposition. Nous ne cherchions pas à étudier les modes d'appréhension de l'ordinateur par l'enfant; nous voulions voir et entendre les élèves en train d'apprendre à travailler -ensemble- avec des ordinateurs. D'autre part, nous ne cherchions pas à évaluer le degré de conformité des usages aux prescriptions pédagogiques de l'école ou du système scolaire : nous cherchions à observer des comportements, non des "résultats". Pour nous, les silences et les coups de folie, les hésitations et les exaltations se valaient bien. Ce positionnement ethnographique et théorique nous a amené à concevoir les NTIC en milieu scolaire moins comme des vecteurs de transmission de savoir (c'eût été là une vision "informationnelle" de la communication) que des modes de reformulations des relations interpersonnelles (en accord avec la définition "intégrative" de la communication, selon Birdwhistell". Tel un système qui doit retrouver un équilibre après introduction d'un nouvel élément, la classe qui "adopte" un ou plusieurs ordinateurs

1 Le projet d'observation, dénommé "Mailbox", est financé par la DG XXII de la Commission Européenne dans le cadre du programme Socrates Open and Distance Learning (ODL). Les partenaires européens du Laboratoire d'Anthropologie de la Communication de l'Université de Liège sont : Atelier (Italie); Consultancy in Distance Education - CIDE (Grande-Bretagne) ; Nasjonalt LaeremiddelSenter-NLS (Centre National pour les Ressources Éducatives - Norvège); le Centre de Recherche Psycho-pédagogique de la Direction Générale du Cycle d'Orientation CRPP-DGCO (Suisse) ; le Centre d'Informatique Pédagogique-CIP (Suisse) ; le Laboratoire de Recherche du Centre National d'Enseignement à Distance et le Laboratoire de Recherche sur l'Industrie de la Connaissance du CNED (France). Selon un accord passé entre nous, l'ensemble des données est exploitable par chacun des partenaires, sans exercice d'un droit de propriété intellectuelle sur les données recueillies. Pour de plus amples infromations sur les observations du projet Mailbox, consulter le site : http://tecfa.unige.ch/tecfa/research/s-mailbox/socratesMailbox-exprmt.html.

2 R. Birdwhistell, Kinesics and Context : Essays on Body Motion Communication, Philadelphia, University of Pennsylvania Press, 1970, p. 86-87. 
doit construire de nouveaux rapports : entre élèves, avec les enseignants, avec les partenaires extérieurs.

Nous avons donc posé qu'il était possible de rompre avec la conception des NTIC en milieu scolaire comme "outils de transmission de savoirs". Nous avons changé de cadre, comme disaient Bateson, Watzlawick et Goffman, et nous avons découvert une autre "réalité" -qui mérite qu'on s'y intéresse ne fût-ce que parce qu'elle permet de fragiliser les certitudes que la vision dominante sur les NTIC en milieu scolaire impose à trop bon compte.

\section{La petite école sur la colline}

Depuis la gare -en fait, un quai le long de la voie unique Bologna-Porretta-, il suffit de traverser le passage à niveau et de monter le long de la route qui s'éloigne de la bourgade. Quand on se retourne, la fumée blanche de l'usine de pâte à papier installée à l'orée de la vallée rappelle à chacun que c'est grâce à cette industrie que la vallée survit. Les montagnes aux alentours sont bien jolies, mais elles ne nourrissent plus grand monde. L'école apparaît au détour du premier virage : parking hésitant, murs de crépi ocre défraîchis, cour de récréation en terre mêlée à du gravillon.

Ce jour-là, Roberta, la maîtresse d'anglais, entre dans la classe de quatrième en brandissant une liasse de feuilles de papier. Les élèves sont en train de terminer l'exercice qu'elle leur a donné pour qu'ils s'absorbent quelques instants tandis qu'elle imprimait leur correspondance électronique dans la minuscule salle des ordinateurs (de l'ordinateur). L'excitation des enfants à la vue des messages devient difficile à contrôler. "Continuez votre exercice", demande Roberta, qui remplit les "boîtes aux lettres" des enfants dans le fond de la classe (il s'agit d'un grand panneau sur lequel chaque élève a collé sur trois côtés une feuille décorée à son nom). Les enfants obéissent mais ne peuvent s'empêcher de tourner la tête pour essayer de voir s'ils ont reçu un message. "Avez-vous fini votre exercice?", demande Roberta. "Ouiii !". "Alors allez voir votre boîte aux lettres. Vérifiez si vous avez reçu un message de votre ami, ramenez-le à votre table, collez-le dans votre cahier et essayez de le comprendre". Hurlements de joie, course folle à travers la classe, sauts par dessus les tables. Roberta laisse faire. Ceux qui n'ont rien reçu retournent lentement à leur place, le visage fermé. Ceux qui ont reçu une lettre 
exultent ; ils bondissent autour de leur chaise et ne parviennent plus à se rasseoir. Debora montre à tous en riant le "Dear Deborah" qu'elle a reçu -avec un h à l'anglo-saxonne.

En fait, peu de lettres sont arrivées. Donato, l'enseignant italien qui correspond avec Roberta depuis Manchester, travaille autrement qu'elle. Au lieu d'inciter les élèves à traduire, avec l'aide d'un dictionnaire, les messages qu'ils reçoivent d'Italie, il écrit une traduction en anglais de chaque message. Il s'ensuit qu'il ne parvient pas à suivre le rythme et que ses élèves répondent avec un certain retard.

Les élèves de Roberta, quant à eux, s'activent à traduire les lettres reçues de Manchester. Un gros Garzanti italien-anglais/anglaisitalien trône sur le bureau de la maîtresse. Les enfants viennent le consulter de temps à autre. Lorsqu'ils ne parviennent pas néanmoins à comprendre leur message, ils demandent l'aide de Roberta. Celle-ci ne leur offre pas de traduction toute faite ; elle les guide plutôt jusqu'au sens général du texte. Par ailleurs, elle propose différents exercices à ceux qui n'ont pas reçu de lettre. Ainsi, Denis est invité à réaliser un grand poster pour rappeler à chacun la nouvelle adresse électronique de l'école de Manchester. "Tu prends toutes les couleurs que tu veux !". Pietro va trouver Roberta et lui demande, sur un ton mi-impatient, mi-désespéré, quand une lettre arrivera pour lui.

Giulia est la première à terminer le décryptage de son message. "Je peux aller à l'ordinateur ?", demande-t-elle avant de bondir hors de la classe. Elle va frapper à la porte de la salle de cinquième, là où se trouvent les trois ordinateurs accessibles aux enfants (mais sans accès direct Internet). Patrizia, l'enseignante de mathématiques ${ }^{1}$, la laisse entrer et continue sa leçon. Ni l'institutrice, ni ses élèves, ne font attention à la petite, qui commence à s'activer autour de l'ordinateur.

L'ordinateur ne s'active pas -il est comme mort. Giulia se tourne vers la maîtresse : "Patti !" Patrizia se tourne vers elle et lui demande, sans la moindre manifestation d'irritation : "As-tu fait tout ce qui était nécessaire ?" Giulia se fige sur place et se frappe la tête : "la fiche !" Elle branche 1'ordinateur, l'allume et entame une série d'opérations. Rien ne se passe vraiment. "Si tu as un problème, tu peux interrompre ma leçon", lui dit Patrizia. Giulia préfère se débrouiller toute seule :

1 Depuis quelques années, nombre d'écoles primaires italiennes ont choisi d'expérimenter la méthode "modulaire" : les enseignants se répartissent le travail par matière et passent d'une classe à l'autre, (presque) comme à l'école secondaire. 
elle prend les feuilles d'instructions (écrites en caractères très colorés par les enfants eux-mêmes), les lit et répète une série d'opérations... Oui ! Elle est dans le système... Elle est dans le programme. Elle commence à écrire. En tapant l'adresse, elle laisse un espace entre les lettres. Patrizia, qui s'est approchée d'elle en silence, lui dit d'une voix calme : "Je pense qu'il y a un problème dans l'adresse". Giulia semble toute étonnée. "L'espace, tu as laissé un espace" -Patrizia lui montre du doigt sur l'écran. "Ces ordinateurs sont vraiment stupides" -s'exclame Giulia en riant.

"Dear Deborah,

Io sto bene..."

Entre-temps, Debora est arrivée. Elle active son ordinateur, mais oublie d'allumer l'écran. Giulia commence à rire. Elles se taquinent tout en continuant à écrire. Debora cherche l'inspiration en louchant sur l'écran de sa voisine. Giulia est complètement absorbée par sa tâche : elle écrit abondamment, avec fluidité et sans fautes d'orthographe. "Aujourd'hui il y a une chercheuse de l'Université de Liège, qui parle italien "abbastanza bene, é molto carina e abbastanza simpatica". Giulia passe ensuite au beau cadeau que son petit ami de cinquième lui a offert pour la Saint-Valentin. "Je te donne mon numéro de téléphone, comme ça tu peux m'appeler" (de Manchester !)

Une troisième élève, Mara, rejoint Debora et Giulia, mais elle doit attendre que l'une d'entre elles ait terminé parce que le troisième ordinateur aujourd'hui ne veut pas démarrer. "Patti, pourquoi ma lettre est-elle si courte ?" -se désole-t-elle.

Giulia a achevé son message, mais elle ne se rappelle plus comment le sauver. Debora l'aide spontanément. Giulia laisse ensuite l'ordinateur à Mara, qui exulte: "Youpee! L'ordinateur blanc !". Tous les ordinateurs sont blancs, évidemment, mais chacun possède son propre système d'opération, signalé par une pastille blanche, rouge ou bleue. D'où un livret d'instructions particulièrement coloré.

Mara demande à son correspondant de lui décrire la tradition du carnaval en Angleterre. "Comment t'habilles-tu ? Vas-tu quelque part à une fête ?" Elle lui décrit ensuite les différents jeux qu'elle va organiser avec ses copines lors de la soirée de carnaval. Denis rentre en classe, en tenant fièrement son poster à bout de bras. Il est suivi par Roberta. Les deux électriciens qui sont par hasard dans l'école ce jour-là sont immédiatement réquisitionnés par Patrizia et Roberta pour accrocher le poster très haut sur le mur. Le bruit d'une Black \& 
Decker remplit aussitôt la classe -mais la leçon de math' continue, imperturbable. Elle se poursuit aussi malgré la procession des élèves de quatrième, qui ne s'arrête qu'avec la sonnerie de la récréation. Quelques enfants filent vers la cour. Mais une dizaine d'enfants restent rassemblés autour des ordinateurs. Sur l'écran de l'une des deux machines, un jeu vient d'apparaître. Sur l'autre, Lorenza est en train d'écrire une lettre à son correspondant. Claudia, une élève de cinquième, la guide à travers la procédure. Sont-ils encore en classe ou déjà en récréation?

\section{Chaos et création}

Lorsque l'"ethnographe" entre dans cette école pour la première fois et essaie de se faire "tout petit" pour observer ce qui s'y passe, il ne peut qu'être surpris par le volume de bruit, par la liberté de mouvement dont semblent jouir les enfants, par l'apparente aisance des enseignants à évoluer dans cet univers. Il a le souvenir de bancs bien alignés, de bras croisés et de punitions. Il sait que l'école primaire a beaucoup évolué au cours des deux dernières décades, tout particulièrement en Italie, où la pédagogie Montessori a eu beaucoup d'influence. Il sait aussi que les instituteurs et institutrices en Italie sont souvent de vrais militants -sinon ils auraient déjà quitté ce métier très mal payé. Mais quand même, les scènes qu'il découvre le surprennent et ne manquent pas de l'amener à se poser de multiples questions : cette école est-elle unique en son genre ? quelles propositions généralisantes va-t-il pouvoir en tirer? l'introduction des ordinateurs dans l'école a-t-elle transformé celle-ci, tant dans son organisation que dans sa pédagogie?

Au fil des semaines d'observation presque participante au fond de la classe de quatrième, l'anthropologue de la communication en vient à formuler les réponses suivantes-elles ne sont pas encore définitives, mais les données qui continuent à s'accumuler les confirment encore.

Tout d'abord, la correspondance électronique entre les élèves de différents pays ne suscite guère d'échanges approfondis : les enfants parlent avant tout d'eux et de leur environnement immédiat ; ils ont souvent beaucoup de peine à remplir quelques lignes. La correspondance électronique ne les incite pas à mieux écrire, à maîtriser l'orthographe, ou même à se sentir à l'aise dans une langue étrangère. 
Les enfants développent des stratégies efficaces mais minimalistes pour écrire et envoyer leurs messages : juste les mots qu'il faut, tapés dans une syntaxe "basique" et envoyés selon une procédure vite routinisée. L'essentiel est ailleurs; les enfants retirent un plaisir fou de l'envoi et de la réception de messages -peu importe ce que ceux-ci disent. C'est le fait d'en recevoir, et dès lors d'en renvoyer pour maintenir le cycle en activité, qui semble fondamental. Recevoir, rendre : il suffit d'ajouter "donner" pour retrouver la célèbre trilogie des obligations définissant le don/contre-don chez Mauss ${ }^{1}$. Sans doute l'observateur ethnographique des usages des NTIC en milieu scolaire ne doit-il pas trop vite chercher à retrouver un des grands principes fondateurs de sa discipline. Mais il semble manifeste à l'observateur attentif des pratiques que les NTIC mobilisent des compétences sociales au moins autant que des compétences cognitives, en ce sens que les enfants ne sont jamais seuls face à l'ordinateur, qu'ils s'organisent entre eux pour maîtriser celui-ci et que le plaisir qu'ils en retirent repose moins sur le contenu du message émis ou reçu que sur la relation que le message permet de créer et d'entretenir.

La dimension rituelle du rapport aux NTIC en milieu scolaire est la deuxième constatation que l'anthropologue de la communication est tenté de faire. D'une part, on vient de le voir, les enfants semblent se comporter dans leur pratique de l'e-mail comme s'ils échangeaient des coquillages dans un système de "kula" -pour le dire très vite. D'autre part, l'enseignante, qui a compris, au moins intuitivement, ce dont il est vraiment question dans le rapport des enfants à l'e-mail, en tire des "événements" (la distribution quelque peu spectacularisée des messages dans les boîtes aux lettres), auxquels elle fait participer chacun (y compris les électriciens de passage). Elle définit ainsi un environnement informatique qui est de l'ordre de la fête, de la surprise, de la (ré)création. On peut parler de rituel pédagogique en ce sens que toutes les activités menées dans le contexte NTIC contribuent à renforcer un sentiment d'appartenance à une petite communauté, élèves, enseignants, personnel technique confondus.

Cette redéfinition des genres, des statuts et des espaces dans un contexte scolaire caractérisé par la présence de NTIC constitue le troisième domaine où l'œil de l'anthropologue trouve à s'exercer. Quand des élèves de dix ans passent librement d'une salle à l'autre

1 M. Mauss, "Essai sur le don", L’Année Sociologique, seconde série, tome I, 19231924, repris dans Sociologie et anthropologie, Paris, PUF, 1950, p. 143-279. 
(mais après avoir demandé la permission et frappé à la porte), quand ils se sentent assez à l'aise pour non seulement appeler l'institutrice par son prénom (pratique aujourd'hui assez courante dans les écoles primaires italiennes) mais pour l'interrompre dans une leçon avec une autre classe, quand ils restent devant leur écran au moment de la récréation et rendent ainsi très visible la disparition de la distinction fondamentale entre temps du travail et temps du jeu, c'est que "quelque chose" s'est passé dans cette école. Non que les NTIC aient provoqué ce bouleversement -du moins rien ne permet d'étayer la thèse d'une telle relation causale-, mais elles l'ont sans doute renforcé et légitimé. Les enseignants sont jeunes et très qualifiés ; ils veulent faire bouger les choses, malgré la faiblesse des moyens dont ils disposent. Les quelques ordinateurs qu'ils reçoivent deviennent l'instrument dont ils vont se servir pour ouvrir les classes, lancer des projets interdisciplinaires, favoriser l'autonomisation des élèves. Le prix qu'ils doivent payer pour ainsi changer l'école est lourd: "agitation" permanente, attention multi-centrée, gestion d'espacestemps sans frontières précises. Mais les résultats sont étonnants. Non que les élèves soient des champions du clavier ; ils sont avant tout des enfants heureux et fiers d'être à l'école, là-haut sur la colline...

\section{Portée et limite d'une lecture "orchestrale" des NTIC}

Dans une perspective "télégraphique", il ne se passe pas grand chose dans cette école. Un chercheur formé dans cette tradition n'y verrait sans doute qu'un joyeux tohu-bohu -il serait d'ailleurs étonnant qu'il s'y rende plusieurs semaines d'affilée : la méthodologie généralement couplée à la vision $\mathrm{Em} / \mathrm{Rec}$ est plutôt quantitative (questionnaires envoyés aux acteurs ou remplis sur place par un "enquêteur"). Le premier apport d'une lecture "orchestrale" des NTIC est celui de la "patience et longueur de temps" que s'impose le chercheur immergé dans son objet; ce n'est qu'à ce prix que le tohubohu disparaît pour laisser place à des "patterns", c'est-à-dire à des régularités, à des prévisibilités, à des "règles". Mais c'est ici aussi qu'apparaît la première limitation d'une telle approche. Rares encore sont les recherches sous contrat qui permettent de travailler lentement, par immersion et décantation. Il faut souvent produire vite, pour des raisons budgétaires notamment, sans laisser le temps au temps. Seules 
les thèses permettent de consacrer douze ou dix-huit mois au "terrain" et un temps équivalent à l'écriture.

Si l'anthropologue de la communication veut se donner les moyens de procéder avec lenteur, c'est aussi parce que la communication ne se résume jamais pour lui à un échange de messages entre un émetteur et un récepteur, et encore moins au contenu des messages échangés. Il veut pouvoir repérer le contexte au sein duquel les acteurs participent à la communication, c'est-à-dire à un processus permanent de confirmation des relations interpersonnelles. Ainsi, dans le cas d'un travail en milieu scolaire, une salle de classe s'impose en général comme le site "naturel" d'observation; mais le contexte pertinent consistera peut-être en un lieu incorporant plusieurs classes, la cafétéria et la cour de récréation. On a vu comment, dans la petite école italienne décrite plus haut, les élèves se déplaçaient librement d'une classe à l'autre, sans déranger les leçons en cours, sur la base d'un consensus entre enseignants. L'approche anthropologique des NTIC ne se réduit jamais aux NTIC et à leurs manipulations immédiates. Les NTIC sont replacés dans différents contextes emboîtés, non seulement le contexte de l'école, son organisation administrative et spatiale, mais aussi son climat pédagogique et son personnel. Cette formulation entraîne sa propre limite : où s'arrêter dans la contextualisation ? C'est l'objet d'un débat sans fin entre "micro-ethnographes" et "macro-ethnographes", les premiers insistant sur la pertinence d'une limitation au contexte de la salle de classe (où les activités sont souvent filmées et analysées), les seconds insistant sur la nécessité d'un long "zoom arrière" allant jusqu'à inclure les activités en famille et dans la communauté1. Pour notre part, nous cherchons à rester simplement "réalistes"; sans nous enfermer dans une seule classe, nous n'avons pas non plus cherché à nous insérer dans les familles des élèves afin d'y observer leurs éventuelles pratiques extra-scolaires des NTIC : trop d'ambition nuit...

De cette réflexion sur l'étendue des mises en relation dans le cadre d'une recherche ethnographique sur les NTIC, on peut retirer un troisième apport de la lecture orchestrale de la vie sociale, qui a trait à sa nature systémique. Il faut ici rappeler la célèbre phrase de Birdwhistell : "Nothing never hapens" (il n'arrive jamais rien que rien

1 Pour une synthèse de ce débat, voir Y. WINKIN, "L'ethnographie en éducation aux États-Unis : une première cartographie", in M.-F. DEGEMBE, Odyssée dans un archipel scolaire, Namur, Éd. Erasme, 1992, p. 129-152. 
n'arrive). Ce qui signifie que l'observateur prend en charge une multitude de comportements, qui seraient éliminés dans une perspective "télégraphique" (sans doute ne seraient-ils même pas relevés ou consignés par l'enquêteur). Par exemple, le repérage du mouvement du bras de l'institutrice au moment où elle revient en classe avec les lettres destinées aux enfants conduit l'observateur à mettre ce geste en relation avec sa demande formelle ("Continuez votre exercice !") et son activité réelle (glisser les feuilles dans les boîtes aux lettres, sans surveiller les élèves). Émerge ainsi l'idée qu'elle agit comme si elle jouait avec eux à dissimuler un cadeau, tel un bouquet de fleurs faussement caché derrière le dos. D'où la suggestion d'un comportement "rituel" de la part de l'enseignante, solennisant par exagération et décalage l'arrivée des messages électroniques.

L'observation d'un mouvement un peu "curieux" a conduit progressivement à une hypothèse organisant un ensemble de "petits faits et gestes" : c'est là le mouvement classique du travail ethnographique en anthropologie de la communication. Mais cette démarche trouve sa limite dans le fait que le repérage d'indices pertinents et leur mise en relation est une affaire d'intuition et de sensibilité personnelle et professionnelle. Ainsi, le mouvement du bras de l'institutrice était "curieux" pour qui, par rapport à quoi ? Une longue anamnèse pourrait sans doute expliquer pourquoi le chercheur a eu, à cet instant précis, le regard attiré par ce geste et l'a noté dans son carnet. Comment, en d'autres termes, son habitus ethnographique a été convoqué en une fraction de seconde. Mais là n'est pas l'essentiel. Il faudrait plutôt pouvoir répondre à la critique selon laquelle la communication orchestrale et la démarche ethnographique qui lui sied bien, ne peuvent faire l'objet de cet enseignement systématique qui à la fois les démocratiserait et renforcerait leur légitimité scientifique. Sans doute, peut-on rétorquer que l'art de voir s'apprend, à la fois sous la guidance d'un maître et grâce à la lecture d'ouvrages d'inspiration ethnographique, tels ceux d'Erving Goffman. Reste que l'anthropologie de la communication -comme d'ailleurs l'anthropologie toute entière- restent mal à l'aise face à cette exigence de transmissibilité intégrale. Mais nous commençons à sortir de notre propos. Il faut revenir pour conclure à la question qui a fondé notre réflexion : le recadrage "orchestral" des NTIC permet-il de sortir de 1" "utopie" de la communication? 


\section{Brouiller les cartes pour mieux refléter le territoire}

L'utopie de la communication repose sur une quête de la transparence et de l'harmonie ; il lui faut donc un modèle de la communication très simple, où des messages circulent dans des conduits mis en réseaux. Pour le dire avec les mots de $\mathrm{Ph}$. Breton :

La communication, sous sa forme utopique, est un univers simplifié, étroitement dichotomique où l'information n'a pas d'autre alternative que l'entropie ${ }^{1}$.

Lorsque les NTIC sont pensées de manière linéaire, selon le schéma de la communication dérivé de Shannon et Weaver ${ }^{2}$, elles se déploient effectivement dans un univers "étroitement dichotomique": c'est l'information ou la mort. Hors l'information, point de salut. Cette alternative est particulièrement vive dans le monde des NTIC pédagogiques en raison d'une équation entre information et savoir : accéder (très rapidement) à l'information, c'est (déjà) savoir. Il suffit de parcourir le gros volume des actes du colloque School Education in the Information Society: Open Classroom II Conference (17-19 September 1997), placé essentiellement sous l'égide de la Commission européenne, pour s'apercevoir de la force institutionnelle de ce paradigme. L'utopie de la communication a encore de beaux jours devant elle, du moins dans le monde de l'éducation. Il faut donc lui opposer quelque résistance.

Il nous paraît que si les NTIC, notamment en milieu scolaire, sont pensées "orchestralement" et sont étudiées ethnographiquement, in situ, en acte -et non plus seulement sur la base de déclarations d'intention et de rapports d'enquêtes (du style survey)- une possibilité leur est donnée de sortir de la communication utopique. Pour trois raisons.

Tout d'abord, parce qu'il s'agit d'une pensée complexe, en rupture avec le sens commun. Ainsi, la notion de participation à la communication n'est pas simple à saisir ; elle oblige à dépasser le cadre de l'interaction (individu-individu ou individu-machine) pour atteindre les dimensions collectives et rituelles des processus communicationnels ; elle suggère d'autres spatialités et temporalités, situées au-delà de l'ici-et-maintenant. Le territoire est complexe ; la

1 Ph. BRETON, op. cit., p. 136.

$2 \mathrm{Cl}$. Shannon et W. Weaver, op, cit. 
carte qui en rendra compte le sera aussi : c'est là le sens du message que l'analyse anthropologique des NTIC propose. Quelques schémas faits de flèches et de boîtes ne peuvent plus rendre compte de la réalité du monde.

Si les NTIC peuvent échapper à la transparence de l'utopie, ce sera aussi parce qu'elles auront été arrachées à l'analyse des contenus qu'elles véhiculent et rendues à l'analyse des contextes qui rendent ces circulations possibles ou impossibles. Tout particulièrement dans le monde de l'éducation, les NTIC sont encore trop souvent présentées comme des cafétérias ou des stations-service où les élèves n'ont plus qu'à s'alimenter. La pensée orchestrale permet d'insister sur la notion de "relation", par opposition à celle de "contenu". Quand bien même le contenu d'un échange de messages électroniques entre un écolier italien et un écolier anglais est assez "nul" informationnellement parlant, la relation qui se noue ne le sera jamais. Les deux enfants apprennent à "donner, recevoir, rendre" : ce sont les bases de la vie en société. Lutter contre l'emprise de l'utopie de la communication sur les NTIC ne consiste pas à minimiser le rôle sociétal de celles-ci ; au contraire, il s'agit de montrer, à partir d'études très concrètes, comment les NTIC contribuent à l'instauration d'une autre dynamique dans différents environnements de travail collectif -mais il ne s'agit jamais de leur donner le rôle de Merlin l'Enchanteur.

Le travail de terrain reste enfin la meilleure garantie contre l'utopie ; il permet de faire remonter à la surface des données trop aisément secondarisées, mais il n'interdit pas l'élaboration conceptuelle, selon le principe de la "grounded theory"1. Ce travail doit déboucher sur un discours écrit, qui peut prendre la forme d'un récit, à la manière de nombre d'anthropologues contemporains (la collection "Terre humaine" chez Plon en est le lieu d'accueil le plus connu en France). Comme le dit bien Philippe Descola ${ }^{2}$, rendre sa recherche plus accessible en acceptant de la narrativiser procède d'une "double responsabilité sociale", d'une part à l'égard de ceux qui ont accueilli l'anthropologue et lui ont fait confiance, d'autre part à l'égard de ses propres concitoyens, qui ont indirectement financé ses recherches. Écrire lisiblement sur la complexité du monde social, c'est lutter par la transparence contre l'illusion de la transparence.

1 A. Strauss et B. Glaser, The Discovery of Grounded Therory, Chicago, Aldine, 1967.

2 Ph. Descola, Les lances du crépuscule, Paris, Plon, 1993. 
Il faut veiller au maintien d'une pluralité de modes d'analyse de la communication. Les NTIC en milieu scolaire constituent un des derniers chapitres de l'histoire de celle-ci ; il ne peut s'écrire uniquement selon les schémas d'une certaine pensée unique. 